\title{
HEPATITIS B: INMUNOGENICIDAD DE LA VACUNA RECOMBINANTE CUBANA ANTI-HBV EN TRABAJADORES DE LA SALUD VACUNADOS SIN SEROPROTECCION
}

\author{
ANGELA B. HOYOS (1), VICTORIA RAMIREZ ALBAJES (2), ANTONIO GONZALEZ GRIEGO (3), \\ CARMEN CECILIA TRUJILLO (4), OSCAR JULIAO (5), PATRICIA PRIETO (5), MONTSERRAT NOGUERA (6)
}

\begin{abstract}
Medimos anti-HBVs a 153 trabajadores de la salud del Hospital Infantil Universitario, "Lorencita Villegas de Santos" en Santafé de Bogotá, a quien se les había vacunado contra la Hepatitis $B$ en los últimos 4 años. 124 habían recibido tres dosis (6 con refuerzo), 18 dos dosis y 11 una sola dosis; no tenían títulos el $25 \%, 33 \%$ Y $73 \%$ respectivamente. Estos trabajadores tenían promedios de edad de 40 años. Se vacunaron 32 de estos trabajadores sin títulos con 1 dosis de 20 mgms de la vacuna cubana recombinante y se cuantificaron títulos anti-HBVs antes y 15 días después de la vacunación con método inmuno-enzimático cuantitativo a ciegas; obteniéndose seroconversión en el $81 \%(>\mathrm{UI} / \mathrm{L})$, seroprotección en el $75 \%(\geq 10 \mathrm{UI} / \mathrm{L})$, y $56 \%$ de los individuos tuvieron valores superiores a $100 \mathrm{UI} / \mathrm{L}$. Estos resultados son altamente significativos y dan una alternativa para un grupo de trabajadores de la salud que no ha logrado títulos protectores y plantea un posibilidad para las poblaciones con factores que los cataloguen como "malos respondedores".
\end{abstract}

\section{INTRODUCCION}

En el mundo entero los trabajadores de la salud que son un grupo de alto riesgo,(l $\left.\begin{array}{lllll}1 & 2 & 3 & 4 & 5\end{array}\right)$ han subestimado la magnitud de la problemática de la Hepatitis B a pesar de los trabajos(6 7) que han mostrado que en salud pública es mucho más grave la hepatitis B que el SIDA sin querer restarle importancia a este último (8) debido a su asociación con el antígeno delta, (9 10 11) la hepatitis crónica y el hepatocarcinoma (11 12). Se ha estimado que en el mundo hay 300 millones de portadores crónicos, 25-30\% de los cuales morirán por enfermedad hepática crónica o hepatocarcinoma (7 11). En Colombia, se han realizado trabajos epidemiológicos (13) y de perfil diagnóstico (14) que señalan la importancia de la problemática a nivel nacional como parte del programa de control (15) en cuanto a profilaxis. La vacuna cubana obtenida por vía recombinante y producida en el centro de Ingeniería Genética y

(1) Neonatóloga, Hospital Infantil Universitario "Lorencita Villegas de Santos". Santafé de Bogotá, Colombia. (2) Victoria Ramírez Albajés, M. D. Centro de Ingeniería Genética y Biotecnología (ClGB). La Habana, Cuba. (3)Antonio González Griego, M.D. Ph. D. Instituto Superior de Ciencias Médicas (ISCM). La Habana, Cuba.

(4) Carmen Cecilia Trujillo, Bacterióloga. Hospital Infantil Universitario "Lorencita Villegas de Santos". Santafé de Bogotá, Colombia.

(5) Oscar Juliao M.D. M.S.P. Asesor de Epidemiolgía, Instituto Nacional de Salud. Santafé de Bogotá, Colombia.

(5) Patricia Prieto, Enfermera Epidemióloga. Hospital Universitario "Lorencita Villegas de Santos". Santafé de Bogotá, Colombia,

(6) Montserrat Noguera. Laboratorios Bio-Lehner S.A. Santafé de Bogotá, Colombia. 
Biotecnología de La Habana se ha estudiado en Cuba con excelentes resultados (16) y también en nuestro medio en el Instituto Nacional de Salud con estudios muy prometedores que potencialmente la ponen como la mejor en imunogenicidad. (17)

\section{MATERIAL Y METODOS}

En Septiembre 1991 se estudiaron 153 trabajadores de la salud del Hospital Infantil Universitario "Lorencita Villegas de Santos" que habían sido vacunados contra la Hepatitis B en los últimos 4 años con esquemas y dosis diferentes para ver su estado de inmunidad con el método de ELISA (Abbott) en el laboratorio del hospital. De las personas que resultaron sin inmunidad, 32 aceptaron entrar en el siguiente estudio: Detección cuantitativa de niveles antes de la vacunación por método de Organon Teknika, vacunación con 1 dosis de 20 fgm de la vacuna cubana recombinate IM en el deltoides y medición de niveles 15 días después de la vacunación. Se utilizó solamente 15 días para estar seguros que la respuesta serológica que se obtenía era inmediata (primaria) que probablemente es la de mejor calidad y duración. Las mediciones de niveles se hicieron simultáneamente (antes y después) en el Instituto Nacional de Salud a ciegas. Los resultados se cuantificaron, se les hizo el estudio estadístico pertinente $\mathrm{X}^{2}$ de series apareadas (18 y se presentan en gráficas y tablas. Se consideró significativo cuando $\mathrm{p}<0.05$.

\section{RESULTADOS}

De los 153 trabajadores de la salud estudiados, 124 habían recibido tres dosis (6 con refuerzo), 18 dos dosis y 11 una sola dosis. El estado inmunitario se describe en la Tabla No. 1. Las características de los 32 trabajadores que entraron al estudio se describen en la tabla No. 2. Vale la pena anotar que de los 32 que por el método de ELISA aparecían como negativos, 2 tenían títulos por el método cuantitativo de Organon Teknika aunque solamente a niveles bajos no protectores.

Como se puede ver también en la tabla No. 2, solo 6 adultos no hicieron seroconversión pasando de $2 / 32(8 \%)$ a $26 / 32(81 \%)$. Para la seroprotección definida internacionalmente como $\geq 10 \mathrm{UI} / \mathrm{L},(19)$
TABLA No. 1

INMUNIDAD DE TRABAJADORES DE LA SALUD ESTUDIADOS

\begin{tabular}{lrrr}
\hline $\begin{array}{l}\text { Número } \\
\text { de dosis }\end{array}$ & Totales & Negativos & Porcentaje \\
\hline & & & \\
3 Dosis más & & & \\
$\begin{array}{l}\text { Refuerzo } \\
\text { 3Dosis }\end{array}$ & 6 & 1 & $16.7 \%$ \\
2 Dosis & 118 & 29 & $24.6 \%$ \\
1 Dosis & 11 & 6 & $33.3 \%$ \\
& 11 & 8 & $72.7 \%$ \\
Total & 153 & 44 & $28.8 \%$ \\
\hline
\end{tabular}

pasaron de (0/32) a $24 / 32(75 \%)$; esta diferencia es altamente significativa con $\mathrm{X}^{2}$ de 36 a 38 para seroconversión y seroprotección respectivamente y $\mathrm{p}<0.0001$. Además se lograron niveles mayores de $100 \mathrm{UI} / \mathrm{L}$ en $18 / 32$, o sea $56 \%$ de los casos (ver gráficas No. 1 y 2).

\section{DISCUSION}

Si tenemos es cuenta que el grupo de personas estudiados no tenían niveles detectables de anticuerpos a pesar de haber recibido un número variable de otras vacunas y además tener un promedio de edad de 40 años podríamos categorizarlos como un grupo "hiporespondedor". El lograr una respuesta de seroconversión del $81 \%$ y una seroprotección del $75 \%$ con una sola dosis estándar (altamente significativo) es evidencia de una buena capacidad antigénica de esta vacuna en estudio. Otra evidencia de efectividad en su capacidad antigénica es la categorización de respondedores con un $56 \%$ de niveles mayores de $100 \mathrm{UI} / \mathrm{L}$ (ver gráfica No. 2). Como conclusión podemos considerar que la vacuna recombinante cubana es una buena alternativa para "hiporespondedores" por lo menos dentro de los trabajadores de la salud y plantea la necesidad de nuevos estudios en otros grupos de población con la misma problemática. 
TABLA 2

\begin{tabular}{|c|c|c|c|c|c|c|c|c|c|c|c|c|c|c|c|c|}
\hline \multirow{2}{*}{\multicolumn{8}{|c|}{ 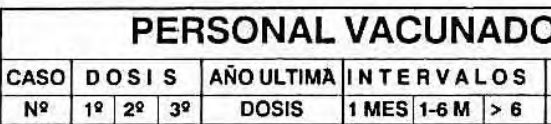 }} & \multirow{2}{*}{\multicolumn{2}{|c|}{\begin{tabular}{c|} 
SELECC \\
TIPO DE \\
VACUNAS
\end{tabular}}} & \multirow[t]{2}{*}{ EDAD } & \multirow[t]{2}{*}{ SEXO } & \multirow{2}{*}{\begin{tabular}{|c|} 
TITULOS \\
anteriores \\
\end{tabular}} & \multicolumn{3}{|c|}{ TITULOS NUEVOS (UI/L) } & \multirow[t]{3}{*}{ PROFESION } \\
\hline & & & & & & & & & & & & & \begin{tabular}{|l|} 
ANTES \\
\end{tabular} & FECHA NUEVA & DESPUES & \\
\hline & & & & & & & & MERK & SPECIA & & & absorvancia & \begin{tabular}{|l|} 
Nov-91 \\
\end{tabular} & VACUNACION & 15 dias & \\
\hline & & & & & & & & & & & & Sep-91 & & & & \\
\hline 1 & 1 & 1 & 1 & 1989 & & 19 & $2^{\circ}$ & & $1,2 \times 3^{\circ}$ & 35 & $F$ & 0.004 & 0 & 18-Nov-91 & 0 & LABORATORIO \\
\hline 2 & 1 & 1 & 1 & 1990 & $1 \times 2^{\circ}$ & & & & $1,2 \times 3^{\circ}$ & 38 & $M$ & 0.017 & 0 & 18-Nov-91 & 0 & LABORATORIO \\
\hline 3 & 1 & 1 & 1 & 1990 & & $1 y z^{a}$ & & $2^{0}$ y $3^{2}$ & 12 & 35 & $F$ & 0.016 & 0 & 19-Nov-91 & 0 & ENFERMERIA \\
\hline 4 & 1 & 1 & 1 & 1989 & $1 \times 2^{\circ}$ & & & 1,2 y $3^{\circ}$ & & 30 & $F$ & 0.019 & 0 & 19-Nov-91 & 0 & LABORATOAIO \\
\hline 5 & 1 & 1 & 1 & 1990 & $1 \times 2^{\circ}$ & & & & $1,2 \times 3^{\circ}$ & 58 & $\mathrm{M}$ & 0.019 & 0 & 18-Nov-91 & 0 & ANESTESIA \\
\hline 8 & 1 & 1 & 1 & 1988 & & $1 \times 2^{\circ}$ & & $2^{2} \mathrm{y}^{2}$ & $1^{0}$ & 40 & $\mathrm{~F}$ & 0.018 & 0 & 22-Nov-91 & 0 & ENFERMERIA \\
\hline 7 & 1 & 1 & 1 & 1989 & & $1 \times 2^{0}$ & & $1,2 \times 3^{\circ}$ & & 42 & $\mathrm{~F}$ & 0.016 & 0 & 18-Nov-91 & 4. & LABOAATORIO \\
\hline 8 & 1 & 1 & 1 & 1989 & 20 & 10 & & & $1,2 \times 3^{2}$ & $\infty$ & $M$ & 0.015 & 0 & 18-Nov-91 & 8 & MEDICO CLINICO \\
\hline 9 & 1 & 1 & 1 & 1990 & & $1 y 2^{2}$ & & $1^{2}$ & $2^{\circ}$ y $3^{\circ}$ & 37 & $F$ & 0.011 & 0 & 20-Nov-91 & 13 & ENFERMERIA \\
\hline 10 & 1 & 1 & & 1988 & $1^{9}$ & & & $10 y z^{0}$ & & 42 & $\mathrm{~F}$ & 0.015 & 0 & 18-Nov-91 & 14 & ENFERMERIA \\
\hline 11 & 1 & 1 & & 1989 & & 12 & & $1^{\circ} \times 2^{2}$ & & 41 & $F$ & 0.062 & 0 & 18-Nov-91 & 37 & LABORATORIO \\
\hline 12 & 1 & 1 & 1 & 1989 & & $1 \times 2^{0}$ & & $2^{2} \times 3^{a}$ & 12 & 43 & $F$ & 0.010 & 7 & 19-Nov-91 & 72 & ENFERMERIA \\
\hline 13 & 1 & 1 & 1 & 1990 & $2^{2}$ & 18 & & & $1,2 \times 3^{\circ}$ & 49 & $F$ & 0.041 & 0 & 19-Nov-91 & 94 & ENFEAMERIA \\
\hline 14 & 1 & 1 & 1 & 1989 & & $1 \times 2^{2}$ & & & $1,2 \times 3^{\circ}$ & 39 & $M$ & 0.041 & 0 & 18-Nov-91 & 98 & MEDICO CLINICO \\
\hline 15 & 1 & 1 & 1 & 1990 & 10 & $2^{0}$ & & & $1,2 \times 3^{\circ}$ & 34 & $\mathrm{~F}$ & 0.044 & 0 & 19-Nov-91 & 154 & ENFERMERIA \\
\hline 16 & 1 & & & 1989 & & & & & $1^{2}$ & 48 & $\mathbf{F}$ & 0.019 & 0 & 18-Nov-91 & 182 & LABORATOAIO \\
\hline 17 & 1 & 1 & 1 & 1990 & & $1 y 2^{\circ}$ & & & $1,2 \times 3^{\circ}$ & 41 & $\mathbf{F}$ & 0.070 & 0 & 24-Nov-91 & 192 & ENFERMERIA \\
\hline 18 & 1 & 1 & 1 & 1989 & & $1^{18}$ & $2^{\circ}$ & $2^{0}$ y $3^{\circ}$ & 18 & 46 & $\mathrm{~F}$ & 0.013 & 0 & 24-Nov-91 & 200 & LABOPATORIO \\
\hline 19 & 1 & 1 & 1 & 1989 & & 10 & 20 & 20 y $3^{\circ}$ & 19 & 43 & $F$ & 0.015 & 0 & 24-Nov-91 & 221 & LABORATORIO \\
\hline 20 & 1 & & & 1989 & & & & & 10 & 38 & $M$ & 0.050 & 0 & 19-Nov-9t & 228 & MEOICO CLINICO \\
\hline 21 & 1 & 1 & 1 & 1989 & & 1 y $2^{\circ}$ & & & $1,2 \mathbf{y}^{2}$ & 25 & $M$ & 0.023 & 0 & 18-Nov-91 & 230 & LABORATORIO \\
\hline 22 & 1 & 1 & & 1989 & & $1 \times 2^{\circ}$ & & $1^{10}$ y $2^{0}$ & & 24 & $F$ & 0.016 & 5 & 18-Nov-91 & 236 & LABORATORIO \\
\hline 23 & 1 & & & 1989 & & & & & 12 & 39 & $M$ & 0.015 & 0 & 19-Nov-91 & 261 & MEDICO ORTOPED. \\
\hline 24 & 1 & 1 & 1 & 1989 & & 12 & $2^{a}$ & $1,2 y 3^{\circ}$ & & 39 & $F$ & 0.012 & 0 & 18-Nov-91 & 264 & LABORATOAIO \\
\hline 25 & 1 & 1 & 1 & 1990 & $1 \times 2^{2}$ & & & & $1,2 \times 3^{a}$ & 48 & $M$ & 0.068 & 0 & 18-Nov-91 & 272 & MEDICO CIRUJANO \\
\hline 26 & 1 & & & 1990 & & & & & 10 & 51 & $M$ & 0.019 & 0 & 22-Nov-91 & 274 & HEMATOLOGO \\
\hline 27 & 1 & 1 & & 1989 & & 10 & & $1^{2} y z^{0}$ & & 28 & $F$ & 0.032 & 0 & 18-Nov-91 & 276 & INMUNOLOGIA \\
\hline 28 & 1 & 1 & 1 & 1989 & & 10 & 2 & $1,2 y 3^{\circ}$ & & 31 & $\mathbf{F}$ & 0.062 & 0 & 19-Nov-91 & 281 & ENFERMERIA \\
\hline 29 & 1 & 1 & 1 & 1989 & $1 \times 2^{a}$ & & & $2^{2}$ y $3^{\circ}$ & $1^{0}$ & 32 & $F$ & 0.016 & 0 & 19-Nov-91 & 302 & LABORATORIO \\
\hline 30 & 1 & & & 1989 & & & & 19 & & 30 & $F$ & 0.014 & 0 & 22-Nov-91 & 304 & LABOPATORIO \\
\hline 31 & 1 & 1 & 1 & 1989 & 1 y $2^{\circ}$ & & & $1,2 \times 3^{2}$ & & 41 & $F$ & 0.050 & 0 & 12-Nov-91 & 307 & LABORATORIO \\
\hline 32 & 1 & 1 & 1 & 1989 & & $2^{0}$ & 10 & 1,2 y $3^{2}$ & & 47 & $\mathbf{F}$ & 0.039 & 0 & 18-Nov-91 & 352 & MEDICO CLINICO \\
\hline
\end{tabular}

GRAFICA No 1

HEPATITIS B

VACUNA CUBANA RECOMBINANTE VACUNADOS SUSCEPTIBLES

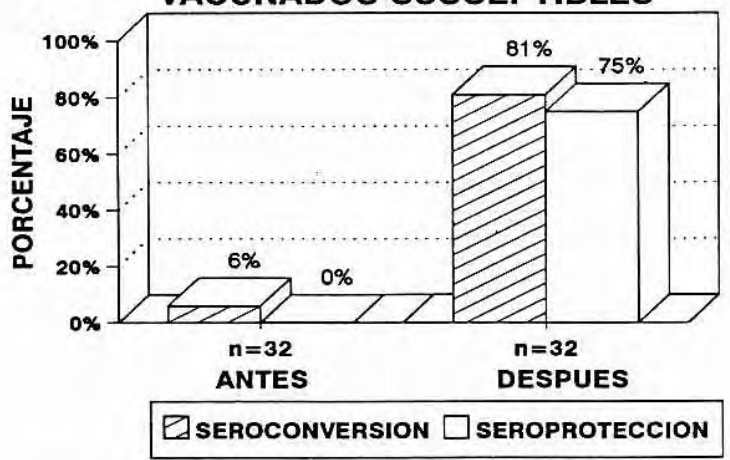

HOSPITAL INFANTIL LORENCITA VILLEGAS DE SANTOS: BOGOTA

\section{GRAFICA No 2}
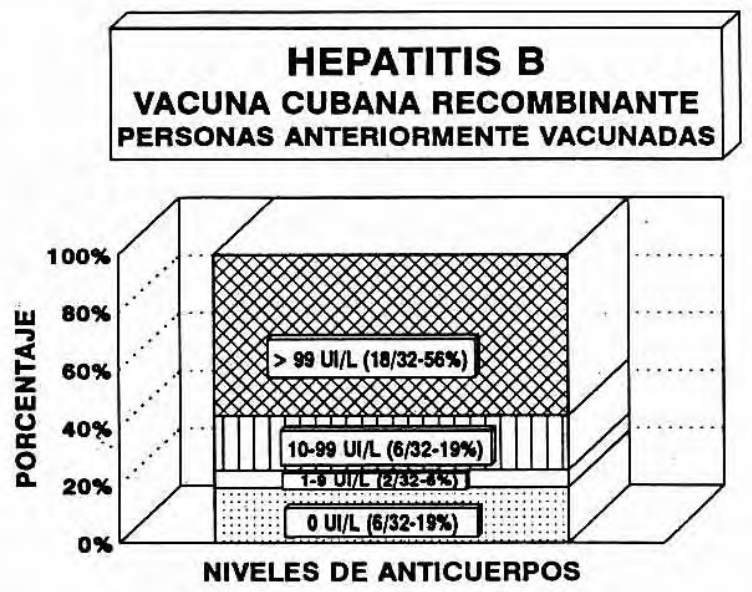

HOSPITAL INFANTIL LOAENCITA VLLEGAE DE EANTOS: BOOOTA 


\section{SUMMARY}

We measured ant-HBs to 153 health care workers in the Children Hospital Lorencita Villegas de Santos in Bogota who had receive Hepatitis B vaccine in the last 4 years. 124 had receive three doses (6 with booster), 18 two doses and 11 only one. They hadn't any positive titles in $25 \%, 33 \%$ and $73 \%$ respectively. Their average age was 40 years old. Then we gave 32 of the worker who has negative titles one $20 \mathrm{mgms}$ dose of the Cuban recombinant vaccine and we measured blind titles before and 15 days after with ELISA quantitative method. $81 \%$ went into seroconversion ( $>0 \mathrm{UI} / \mathrm{L}$ ), $75 \%$ seroprotection $(\geq 10 \mathrm{IU} / \mathrm{L})$ and $56 \%$ had levels over 100 UI/L. This results are hilly significant and raises a possibility for health care workers who are "poor antibody responders" with other types of vaccines or even other population with the same problem.

\section{BIBLIOGRAFIA}

1. Lewis $\mathrm{TL}, \mathrm{Alter} \mathrm{HJ}$ y col. A comparison of the frequency of hepatitis B antigen and antibody in hospital and nonhospital personnal. N Engl J Med. 1973;289:647.

2. Denes $A E$, Smith $J$ y col. Hepatitis $B$ infecction in physicians: remets of a nationwide seroepidemiologic survey.

3. Dienstag JL y Ryan DM. Ocupational exposure to hepatitis $B$ virus in hospital personnel. Infection or immunization? Am J Epidemiol. 1982;115:26.

4. Snydman DR, Munoz A y col. A multivariate analysis of risk factors for hepatitis $B$ virus infection among hospital employees screened for vaccination. Am J Epidemiol. 1984; $120: 684$.

5. Hadler SC. Hepatitis B infection and helth care workers. Vaccine. 1990;8:528 (suppl).

6. Sherlock S. Hepatitis B: the disease. Vaccine 1990; 8 suppl:56.
7. World Health Organization. International conference on the control of hepatitis B in the developing world. Cameroon. Oct 7-9, 1991.

8. Stagno S. Comunicación personal.

9. Rizzetto M, Canese MG y col. Immunofluorescense detection of new antigen-antibody system (delta/anti-delta) associated to hepatitis $\mathrm{B}$ virus in liver and serum of $\mathrm{HBsAg}$ carriers. Gut 1977:18:997.

10. Torres JR, Mandolfi A. Protected outbreak of severe delta hepatitis: experience in an isolated amerindians population of the upper Orinoco basin. Rev Infec Disease 1991;13:52.

11. Zeldis JB y Crumpacker CS. Hepatitis. En: Remington JS, Klein JO. (eds). Infectious diseases of the fetus and newborn infant. Philadelphia, Third ed. WB Saunders Co, 1990. p. 574.

12. Cheng-Po Hu, Li-Chun W y col. Identification of a human hepatocellular carcinoma-associated antigen by a monoclonal antibody. Viral Hepatitis and liver disease. A. J. Zuckerman editor. Alan R. Liss inc. New York 1988 p. 780.

13. Juliao O. Prevalencia de antígeno de superficie en Colombia. Instituto Nacional de Salud. Biomédica Vol. 11, 1991.

14. Buitrago B, Popper H, y col. Specific histologic features of Santa Marta hepatitis: a severe form of hepatitis delta virus infection in northern South America. Hepatology 1986; 6:1285.

15. Juliao $\mathrm{O}$, Ramírez-Albajés $\vee$ y col. Inmunogenicidad de la vacuna recombinante cubana. Biomédica Vol. 11, 1991.

16. Gonzalez-Griego, A. Hepatitis B, vacuna cubana recombinante. Conferencia en el auditorio, Hospital Infantil "Lorencita Villegas de Santos" Bogotá, noviembre 15, 1991.

17. Juliao, $\mathrm{O}$. Estudio de inmunogenicidad para dos vacunas recombinantes contra Hepatitis comparando dos esquemas. Instituto Nacional de Salud. Biomédica Vol. 11, 1991.

18. Colton T. Statistics in medicine. Little Brown and Co. Ed USA, 1974; ps 347, 347.

19. Couroucé $A M$, Jungers $P$ y col. Hepatitis $B$ vaccine in dialysis patients. N Engl J Med. 1984; 311:1515. 